\title{
Alarm system of optical fibre using the thermal-optical sensibility of the PNIPAAm polymer
}

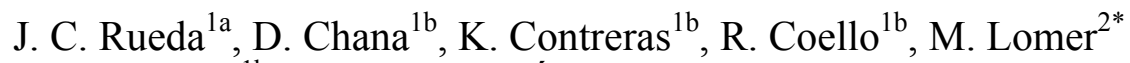 \\ ${ }^{1 \mathrm{a}}$ Laboratorio de Polímeros, ${ }^{1 \mathrm{~b}}$ Laboratorio de Óptica, Departamento de Ciencias, Sección Física, \\ Pontificia Universidad Católica del Perú, Apartado Postal 1761, Lima 32, Perú \\ ${ }^{2}$ Photonics Engineering Group, University of Cantabria, Avda. Los Castros s/n, 39005, \\ Santander, Spain
}

\begin{abstract}
An alarm system as extrinsic sensor on optical fibers for detecting and controlling inflammable liquids based on thermosensitive proprieties of the PNIPAAm hydrogel is presented. The changes on the optical proprieties of the PNIPAAm with the temperature (being its LCST $32^{\circ} \mathrm{C}$ ), induce abrupt changes on the light intensity and they act as an alarm signal, which is transmitted by optical fibers and after they will be processed by an optoelectronic circuit, responsible to active an alarm. An appropriate system consists of the hydrogel connected between its ends to two segments of plastic optical fibers (source and receiver) and they turn on the alarm when a photo detector does not receive light when the hydrogel becomes when it reaches threshold of temperature. The characterization of the hydrogel and the experimental results are presented for a prototype.
\end{abstract}

Keywords: alarm sensing, smart hydrogels, plastic optical fiber.

\section{INTRODUCTION}

Polymers are most promising materials for practical applications in the field of optoelectronics. Special attention has been paid to the polymers that respond to environmental stimulus. These new materials have many fields of application, by example the medical diagnostic and environmental protection including the industrial applications. An example of these "smart materials" is the poly(N-isopropylacrylamide) (PNIPAAm), which presents conformational transitions to low temperatures of critical solution and a remarkable hydration and dehydration under changes in aqueous solution in response to small changes of temperature [1]. In optical sensors, the hydrogels present an interest very attractive for measurements of diverse physical and chemical parameters [2], examples of humidity sensors or $\mathrm{pH}$ sensors have been demonstrated [3]. At other hand, the plastic optical fibers (POF) present a series of advantages with respect to silica fibers. These fibers have grand diameter, high numeric aperture, they also are easy to use, excellent flexibility, and with the use of electronic devices as LED, photodiodes, all that constitute systems of low-cost.

In this paper, it is presented an alarm system employing an extrinsic sensor of optical fiber for detecting and controlling inflammable liquids based on the thermo-responsive proprieties of PNIPAAm polymer. The PNIPAAm polymer is located at a glass capillary in liquid phase and at its ends, it is fixed two segments of POFs. At room temperature, the PNIPAAm is transparent to visible light, it transmits the light around $100 \%$, but when the room that rounds it, increases its critical temperature of $32^{\circ} \mathrm{C}$, it becomes opaque, then the transmitted light decreases. This abrupt change on the light intensity transmittance induces an optical signal for acting such as an alarm. The connected optical fibers at its ends of the PNIPAAm polymer do more easily to sense at distances in aggressive or dangerous environments. We present the characterization on temperature of the PNIPAAm polymer and its results as prototype of the alarm system.

\footnotetext{
*1omer@teisa.unican.es; phone +34-942-201495; fax +34-942-201873; unican.es

Third European Workshop on Optical Fibre Sensors, Antonello Cutolo, Brian Culshaw, José Miguel López-Higuera, Eds., Proceedings of SPIE Vol. 6619, 661946, (2007) $\cdot 0277-786$ X/07/\$18 $\cdot$ doi: 10.1117/12.738672
} 


\section{FABRICATION}

\section{Polymer preparation}

The thermally responsive polymer material is poly( $\mathrm{N}$-isopropylacrylamide). This material is formed by copolymerization of a mixture containing of N-isopropylacrylamide (NIPAAm) and azobisisobutyronitrile (initiator). This process produces polyNIPAAm copolymer. PolyNIPAAm is one of the best-known temperature sensitive polymers. It undergoes a rapid and reversible phase transition form extended hydrated chains (swollen state) below the lower critical solution temperature (LCST) of $32^{\circ} \mathrm{C}$ to collapsed hydrophobic coils (shrunk state) at a temperature above LCST.

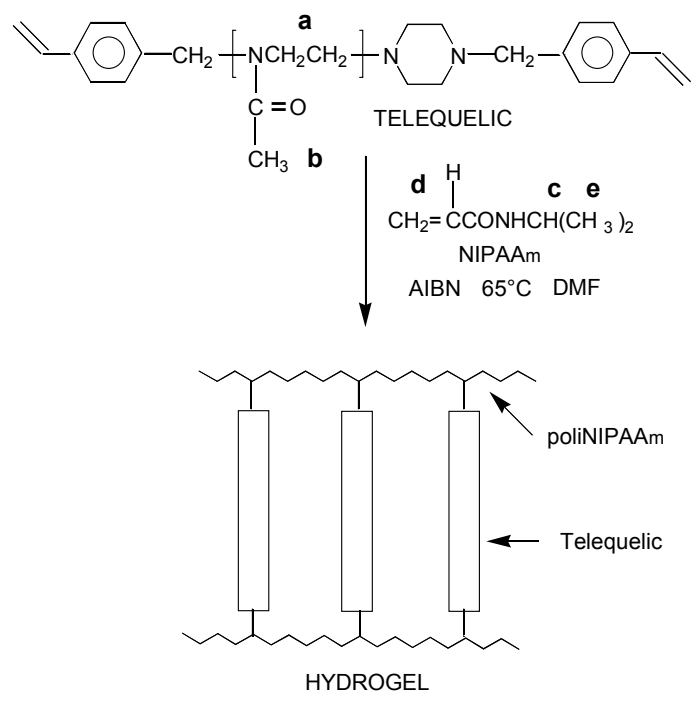

Figure 1. Chemical structure of the hydrogel Poly(NIPAAm) and 2-methyl-2-oxazolines.

The hydrogels are cross-linking polymers in three-dimensional form of natural or synthetic nature. These hydrogels swell in contact with water forming a soft and elastic material; retain a quantity of the water in its structure without dissolve. The solid polymers are especially adequate for forming gels thank to their structure of large chains. The flexibility of these chains permit that these can be deform with the aim of permit the entrance of the molecules of the dissolvent at its three-dimensional structure.

\section{Proprieties of the hydrogel (PNIPAAm)}

The PNIPAAm polymer, because to the aqueous solutions show a conformational transition at critical temperature (LCST) to $32^{\circ} \mathrm{C}$, where it occurs a sudden precipitation of the polymer in all water polymer [4]. This process is reversible because when the temperature of system decreases, the Poly(NIPAAm) dissolves again. The LCST value can to be controlled during the co-polymerization process adding hydrophilic or hydrophobic monomers respectively. The chemical structure of the Poly(NIPAAm) polymer is shown in the fig. 1.

The PNIPAAm polymer in aqueous solution exhibits an LCST at $31-32^{\circ} \mathrm{C}$. Physicochemical phenomena occurring in an aqueous solution of PNIPAAm at the LCST have been the subject of studies carried out using a variety of experimental techniques. It has been demonstrated that the phase separation process of PNIPAAm occurs in two steps. In the first step, an individual polymer chain collapses from an extended coil into a globule. In the second step, an aggregation of the individual globules occur leading to a macroscopic phase separation.

The optical proprieties of the Poly(NIPAAm) polymer show a good transparency at room temperature, exhibiting a refraction index of 1.47 [5]. When the polymer gets the LCST value, this becomes opaque to the incident light intensity, acting as a switch. This characteristic is used for design it as alarm sensor. When the Poly(NIPAAm) is synthesized with 2-methyl-2-oxazoline (MeOXA), the thermo sensibility of the hydrogel can to vary between a range $32^{\circ} \mathrm{C}$ and $75^{\circ} \mathrm{C} \mathrm{[6].}$ 


\section{Device of the sensor}

The optical setup is shown in the fig. 2. The fiber used is a PMMA-POF index step with diameter $2 \mathrm{~mm}$ including the jacket, where core index and cover index are $\mathrm{n}_{\mathrm{co}}=1.492$ and $\mathrm{n}_{\mathrm{cl}}=1.402$ respectively. A source of light LD in wavelength $660 \mathrm{~nm}$ has been used. The transducer head for measuring temperature inside inflammable liquids is based on a closed capsule, where is located the poly(NIPAAm) hydrogel with its ends connected to two optical fibers. The changes of transmitted intensity are detected by the PIN photodiode placed in the fiber ending. When the transducer head is inserted in a tank of inflammable liquids, from which it is necessary to sense the temperature for avoiding explosions, the photodiode registers variations in the transmittance.

All sensor consists of a source, two plastic optical fibers, the transducer head with the Poly(NIPAAm) hydrogel, the detector and the optoelectronics unit. Both the source as the detector are driven by the optoelectronic unit, where the signal is processed for converting the light intensity changes in voltage and after to emit a sound alarm. Both the source and the detector are coupled with the plastic optical fibers. The transducer head is a small ampoule where it is located the PNIPAAm hydrogel.

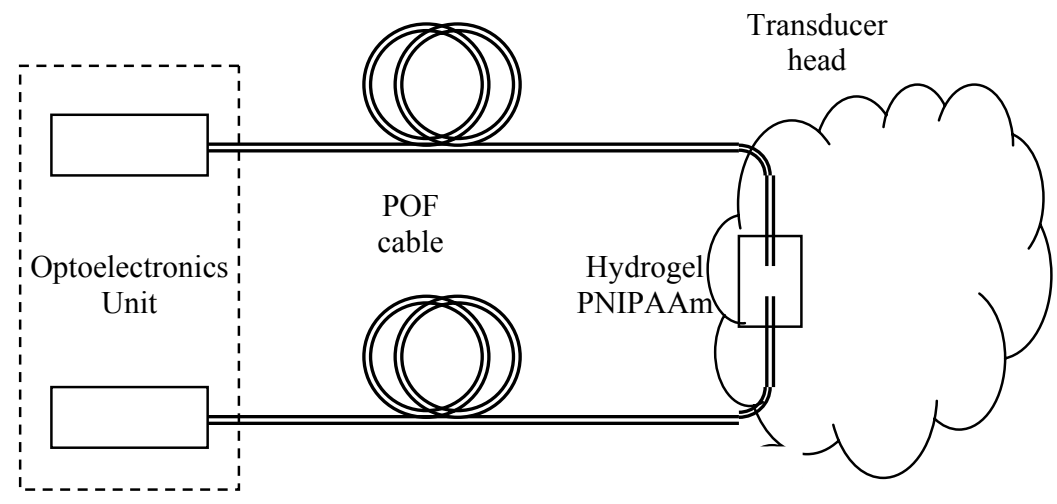

Figure 2. Optical setup for measuring temperature under environmental.

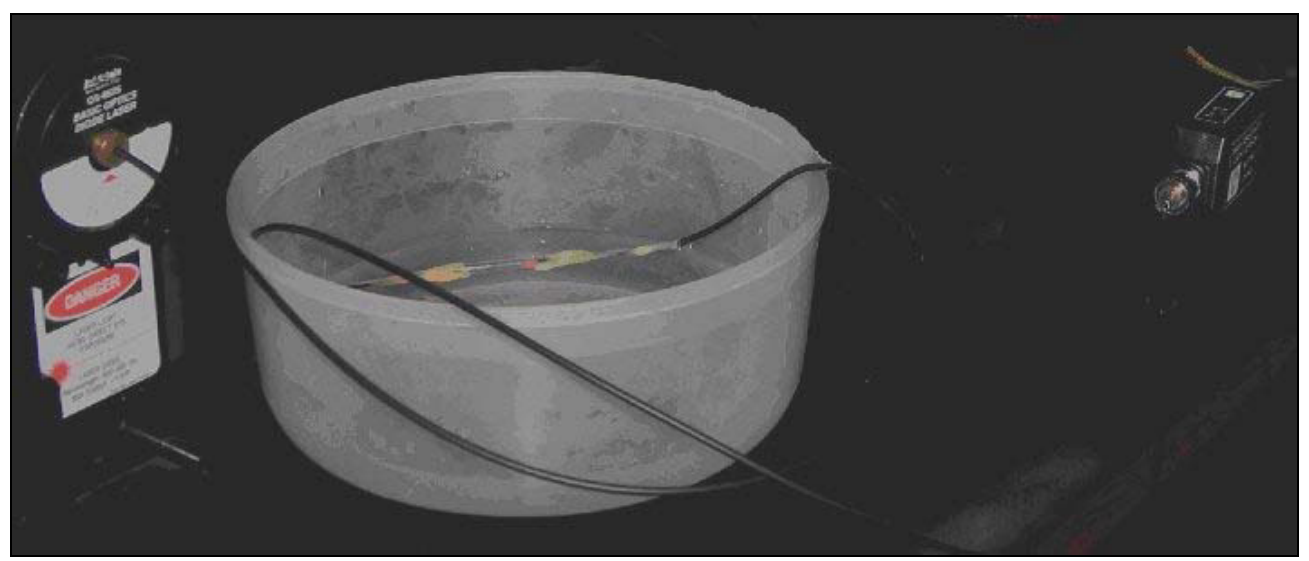

Figure 3. Optical setup at laboratory, where it is shown the light source, the transducer head and the light detector. 


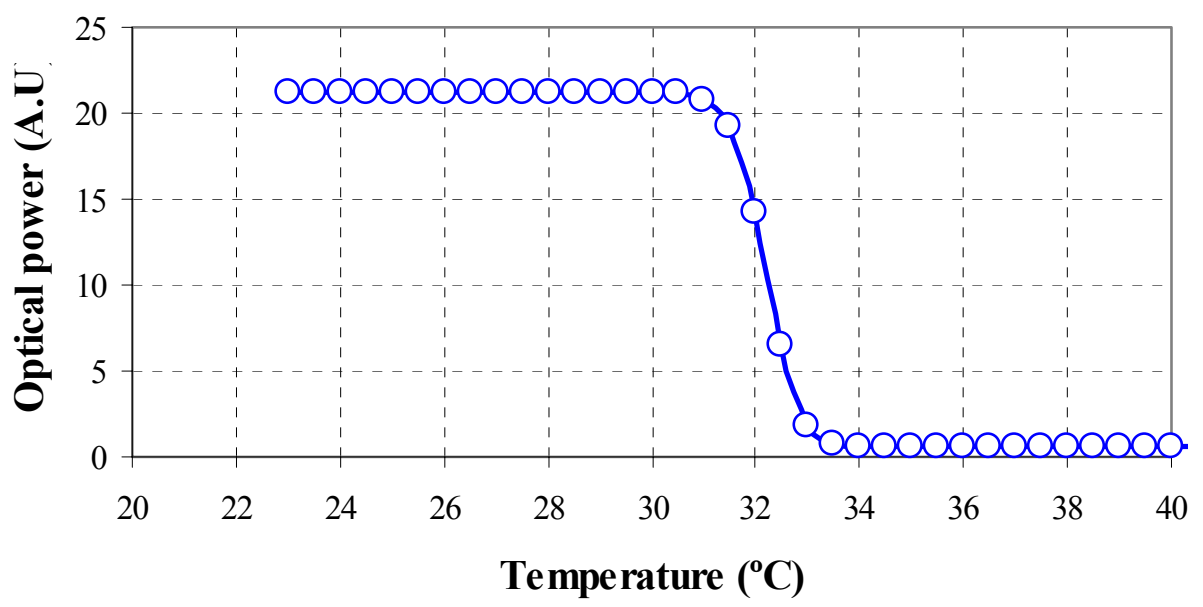

Figure 4. Optical power detected at photodiode versus temperature at the Poly(NIPAAm)

\section{RESULTS AND CONCLUSIONS}

Lower critical solution temperatures (LCSTs) for the polymer samples were determined by estimation of the cloud points from optical power detected at photodiode (turbidity measurements). The temperature of the water-jacketed cell holder was controlled by a water-circulating bath and varied from 22 to $40^{\circ} \mathrm{C}$ at a rate of about $0.2^{\circ} \mathrm{C} . \mathrm{min}^{-1}$. The exact temperature of the sample fluid was measured with a thermocouple. The intensity of scattered light was plotted against the temperature of the solution. The cloud point was determined graphically, as it is shown in the fig. 4 .

\section{CONCLUSIONS}

We have implemented an alarm system based on two optical fibers for sensing inflammables liquids using the thermal sensibility of the PNIPAAm polymer. We have experimentally determined the LCST value of $32^{\circ} \mathrm{C}$, which is the temperature threshold for activating the alarm. Other polymers based in the PNIPAAm, synthesized with different molar ratio between the polyNIPAAm and the MeOXA will permit to vary the thermal sensitivity and their LCST, increasing the use range as alarm system and to open the possibility for other applications. The demonstrated advantages of the POFs are easy to handling, easy to connect to sources and light detectors. All that, make alarm system cheaper.

\section{REFERENCES}

[1] M. Heskins and J. E. Guillet, J. Macromol. Sci. - Chem., A2(8), 1441 (1968).

[2] Bartlett R J Chandy R P Eldridge P Merchant D F Morgan R and Scully P J 2000 Plastic optical fibre sensor and devices Trans Inst Measurement and Control 22 431-457

[3] W C Michie et al 'Distributed pH and Water Detection Using Fibre Optics Sensors and Hydrogels', Journal of Lightwave Technology, Vol. 13, No. 7, p1415-1421, 1995.

[4] H.G. Schild, Prog. Polym. Sci. 1992, 17, 163-249.

[5] Handbook of Chemistry and Physics, CRC Press, 56 Edition, 1975.

[6] J.C. Rueda, ZS. Zschoche, H. Komber, D. Schamljohann and B. Voit. Macromolecules, 2005, 38, 7330-7336. 